\title{
Prescription of a THC/CBD-Based Medication to Patients with Dementia: A Pilot Study in Geneva
}

\author{
Barbara Broers $^{\mathrm{a}}$ Zacharie Patà ${ }^{\mathrm{b}}$ Aline Mina $^{\mathrm{b}}$ James Wampfler ${ }^{\mathrm{c}}$ \\ Christian de Saussure ${ }^{c}$ Sophie Pautex ${ }^{d}$ \\ a Unit of Dependencies, Department of Primary Care and Community Medicine, Geneva University Hospitals, \\ Geneva, Switzerland; bFaculty of Medicine, University of Geneva, Geneva, Switzerland; 'EMS "Les Tilleuls," \\ Geneva, Switzerland; ${ }^{d}$ Department of Palliative Medicine, Geneva University Hospitals, Geneva, Switzerland
}

\section{Keywords}

Cannabinoids · Dementia · Treatment · THC/CBD

\begin{abstract}
Background: Dementia is increasing worldwide. No effective medication is currently available for the treatment of the underlying disease and accompanying behavioral symptoms. Cannabinoids might have a beneficial effect, but clinical studies with (low-dose) synthetic THC have not been conclusive. Objective: To test the acceptability, practical aspects, and clinical outcomes of the introduction of a THC/ CBD-based oral medication in severely demented patients in a specialized nursing home in Geneva. Methods: This was a prospective observational study. Results: Ten female demented patients with severe behavior problems received oral medication with on average $7.6 \mathrm{mg} \mathrm{THC} / 13.2 \mathrm{mg}$ CBD

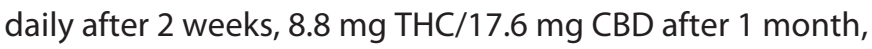
and $9.0 \mathrm{mg} \mathrm{THC/18.0} \mathrm{mg} \mathrm{CBD} \mathrm{after} 2$ months. The THC/CBDbased oil was preferred. Neuropsychiatric Inventory, CohenMansfield Agitation Inventory score, and a behavior problem visual analog scale decreased by $40 \%$ after 2 months, rigidity score by $50 \%$. Half of the patients decreased or stopped other psychotropic medications. The staff appreci-
\end{abstract}

ated the decrease in rigidity, making daily care and transfers easier, the improved direct contact with the patients, the improvement in behavior, and the decrease in constipation with less opioids. There was no withholding of the medication for reasons of side effects, and the effects persisted after 2 months. Conclusions: An oral cannabis extract with THC/ $\mathrm{CBD}$, in higher dosages than in other studies, was well tolerated and greatly improved behavior problems, rigidity, and daily care in severely demented patients.

(c) 2019 The Author(s)

Published by S. Karger AG, Basel

\section{Introduction}

Dementia is a syndrome characterized by a deterioration in memory, thinking, behavior, and autonomy in daily life activities. According to the World Health Organization [1], there were around 50 million persons worldwide suffering from dementia in 2017, and this number is increasing with the global aging of the population in most countries. Dementia has different causes, but Alzheimer disease is the most common form (two-thirds of cases). Approximately 5 out of 6 patients with dementia will develop behavioral and psychological symptoms of

\begin{tabular}{|c|c|}
\hline KARGER & $\begin{array}{l}\text { (c) } 2019 \text { The Author(s) } \\
\text { Published by S. Karger AG, Basel }\end{array}$ \\
\hline $\begin{array}{l}\text { E-Mail karger@karger.com } \\
\text { www.karger.com/mca }\end{array}$ & $\begin{array}{l}\text { This article is licensed under the Creative Commons Attribution- } \\
\text { NonCommercial-NoDerivatives } 4.0 \text { International License (CC BY- } \\
\text { NC-ND) (http://www.karger.com/Services/OpenAccessLicense). } \\
\text { Usage and distribution for commercial purposes as well as any dis- } \\
\text { tribution of modified material requires written permission. }\end{array}$ \\
\hline
\end{tabular}

Prof. Barbara Broers

Unit of Dependencies, Department of Primary Care and Community Medicine Geneva University Hospitals, 6, rue Gabrielle-Perret-Gentil

$\mathrm{CH}-1211$ Geneva (Switzerland)

E-Mail barbara.broers@unige.ch 
dementia (BPSD) during the course of the disease. These disturbances, namely agitation, depression, delusions, and hallucinations, are strongly correlated with each other. Currently, the options for treating BPSD include pharmacological and nonpharmacological therapies. Psychotropic medications are often used to reduce the frequency and severity of BPSD, but in the majority of patients, they provide only modest symptom control and important side effects [2]. The interest of cannabinoids in Alzheimer disease and other forms of dementia has increased first as neuroprotective drugs in animal studies. Indeed, some studies suggest a potential beneficial effect of $\mathrm{CB} 1$ and $\mathrm{CB} 2$ receptor agonists on reducing harmful $\beta$-amyloid peptide action and tau phosphorylation seen in Alzheimer disease as well as increasing intrinsic brain repair mechanisms [3]. Several clinical trials, conducted in patients with severe dementia and BPSD, with one randomized study giving up to $4.5 \mathrm{mg}$ THC daily, demonstrated that this can be safely administered to patients with dementia, but did not demonstrate any effect on BPSD [46]. Recent studies suggest that medications combining THC and CBD might have benefits over pure (synthetic) THC preparations [5].

The aim of this pilot study was to demonstrate the feasibility of the administration of a THC/CBD-based medication in patients with severe dementia and BPSD and/ or rigidity living in one nursing home in Geneva.

\section{Subjects and Methods}

\section{Study Medication}

In Switzerland various cannabinoid-based medications are available, in specialized pharmacies, with an authorization of the Federal Office of Public Health, for a specific patient, indication, and medication and for a limited duration $(6$ months at the time of the study). Available medications are nabiximol (oromucosal spray with THC/CBD in equal amounts), dronabinol solution (pure THC), cannabis tincture ( $11 \mathrm{mg}$ THC/22 mg CBD), and cannabis oil (11 mg THC/24 mg CBD). At the start of the study we decided to use the cannabis tincture, since the nurses had the experience of difficulties in administering the oromucosal nabiximol spray (patient biting on the tip or moving her head so the spraying was random).

The introduction of the medication was according to protocol with the principle of "start low, go slow," stop when sufficient effect, stop medication when no effect or side effects. The medication was given three times daily.

The study setting, "Les Tilleuls" in Geneva, is a nursing home specialized in the care of elderly with severe dementia. The staff includes nurses, nurse-aids, an appointed family doctor, and a psychiatrist. The psychiatrist met with the legal representatives of potential patients to get permission for the prescription of cannabinoid-based medication (from the Federal Office of Public Health) and the participation in the study. He was also responsible for the prescriptions of cannabinoid-based and other psychotropic medications.

\section{Study Design}

This is a prospective observational pilot study of 10 patients who received a cannabinoid-based medication. The inclusion criteria were patients with dementia from different origins (Alzheimer disease, vascular, mixed) with persisting behavior problems (Neuropsychiatric Inventory [NPI] score $>10$ ) notwithstanding optimal conventional treatment and consent obtained from the legal representative. The exclusion criteria included unstable somatic disorders with frequent changes in prescriptions, severe cardiac insufficiency or unstable heart rhythm, symptomatic orthostatic hypotension, receiving tricyclic antidepressants, fluoxetine, or carbamazepine, and change in psychotropic medication in the last week.

\section{Assessments and Instruments}

Patients were assessed before the introduction of the cannabinoid medication, after 2 weeks, 4 weeks, and then every month. The following validated scales $[7,8]$ were used by one trained nurse: (1) NPI: severity of the 12 most frequent psychiatric problems in dementia. (2) Cohen-Mansfield Agitation Inventory (CMAI): severity of agitation. (3) Unified Parkinson Disease Rating Scale (UPDRS), notably item 22, the degree of rigidity with passive movements. (4) Barthel index for daily activities. (5) Visual analog scale (VAS) from 0 to 10 for the most invalidating daily activity (determined by staff). (6) VAS from 0 to 10 for the most invalidating or disturbing behavior (determined by staff). Blood pressure, weight, all medications received, and other observations were noted in the patient file.

\section{Database and Analysis}

Two medical students entered all data in the clinical record files for the study (with crosscheck) using a secured Excel database. Only descriptive results were used for this pilot study, with comparisons up to 2 months of medication intake.

\section{Results}

We included for this pilot study, as planned, 10 severely demented patients with an NPI score $>10$, all females, with an average age of 79.5 years. Inclusion was gradual and follow-up varied from 2 to 5 months.

After a few weeks, we discovered that 3 patients seemed to have pain when swallowing, even worse with the (alcoholic) tincture, and had mouths ulcers. When cannabis tincture was changed to cannabis oil, the ulcers disappeared. We decided to privilege cannabis oil as medication, given with a small piece of chocolate cake to the patients to facilitate intake.

The average daily dosage was $7.6 \mathrm{mg}$ THC/13.2 $\mathrm{mg}$ CBD daily after 2 weeks, $8.8 \mathrm{mg}$ THC/17.6 mg CBD after 1 month, $9.0 \mathrm{mg}$ THC/18.0 mg CBD after 2 months, and 
then remained stable. The lowest daily dosage was $7 \mathrm{mg}$ $\mathrm{THC} / 14 \mathrm{mg}$ CBD, the highest $13 \mathrm{mg}$ THC/26 mg CBD.

One patient died after 1 month for reasons unrelated to the cannabinoid medication. No patient stopped the cannabinoids for reasons of side effects. Systolic blood pressure decreased from 135.4 to $120 \mathrm{~mm} \mathrm{Hg}$, other vital parameters (diastolic blood pressure, heart rate, weight) remained stable over time.

The average NPI score decreased from 71.1 to 38.3 after 2 months, the CMAI score from 74.5 to 47.5. The rigidity score (UPDRS) decreased from 3.4 to 1.7. Scores for daily activities both decreased, but this was due to a decrease (less good functioning) in 2 patients, whereas 7 patients improved. The VAS score for the most invalidating behavior problem (screaming, aggressive behavior, tearing clothes) decreased from 9 to 5 . The effects persisted after 2 months for the 4 persons for whom longer follow-up was available.

The nurses, several of whom were quite reluctant to the study in the beginning, observed in almost all patients less overall rigidity, with more relaxed faces, necks, shoulders, and limbs, making daily washing and transfers easier and more comfortable. They described the patients as calmer, more relaxed, less irritable, and smiling more. Two women with persistent screaming almost stopped doing so, which was a major relief for other patients and the staff. One patient stopped frequent vomiting. Two patients could stop all morphine within 3 months and 1 patient decreased by two-thirds in 2 months. These 3 patients had no more constipation, which was noted as an important time gain for the staff, beyond the benefit for the patients. One patient decreased benzodiazepine use by three-fourths after 3 months and 1 patient stopped two antipsychotic medications after 1 month.

The feedback from the families was astonishingly positive overall. They were in general thankful that the director and the doctor were trying to improve the situation for their relatives, and all accepted to participate in the study. Most were satisfied with the improvement, while a few were disappointed by the limited change, probably due to high expectations.

\section{Discussion and Conclusion}

Our study suggests that a THC/CBD oral medication in severely demented patients with behavior problems is acceptable, well tolerated, and improves rigidity and behavior overall. It allowed the decrease or stop of other psychotropic medications in half of the patients. The staff appreciated the decrease in rigidity, making daily care and transfers easier, the improved direct contact with the patients, and the improvement in behavior. There was no withholding of the medication for reasons of side effects, and the effects persisted after 2 months.

We used a medication with a natural cannabis extract, with a THC/CBD ratio of 1:2. Compared to other studies, we used a higher dosage, with an average of $9 \mathrm{mg}$ THC daily after 2 months versus $2-5 \mathrm{mg}$ in most studies (7 $\mathrm{mg}$ in one study) [3]. Other studies used pure, often synthetic, THC medications; we hypothesize that a natural cannabis extract might be better tolerated and/or that $\mathrm{CBD}$ adds to the overall positive effects on behavior. Still, compared to the average doses used in multiple sclerosis patients (average 7 puffs of nabiximol per day corresponding to $17 \mathrm{mg}$ THC/34 mg CBD), doses were low, but our patients were older (almost 80 years). We privileged the cannabis oil for different reasons: oromucosal spray is difficult to administer in demented patients, and the cannabis tincture (with alcohol) seemed to cause oral ulcers in 3 patients. Cannabis oil has to be given with some oil-rich food; after having tried out different food options, the nurses found that putting the drops on a small piece of chocolate cake was the best administration option.

Doing research in severely demented patients is not easy since patients cannot give an oral feedback and their behavior and symptoms can be influenced by different factors beyond the cannabinoids medication. Other limiting factors of our study are the observational design, the low number of patients included, and the lack of pharmacological data. Also, the increase in cannabis oil and the decrease in other psychotropic medication was based on the clinical judgement of one (experienced) psychiatrist.

Still, to our knowledge this is one of the first clinical studies on the use of a THC/CBD "natural cannabis"based oral medication in severely demented patients, suggesting excellent tolerance, acceptability, and benefit for the patients, notably on BPSD. Former studies often used pure THC, since this was considered the "active" ingredient of cannabis. Basic research suggests that the interest of cannabinoids in Alzheimer disease, and other forms of dementia, goes well beyond THC only [5]. Therefore, we suggest future studies on cannabinoids-based medication to focus more on standardized extracts of natural cannabis with different cannabinoids.

Our study should be repeated in a randomized clinical trial. We are planning to do so after a pharmacological analysis and a longer observation period of our patients.
58

Med Cannabis Cannabinoids 2019;2:56-59 DOI: $10.1159 / 000498924$
Broers/Patà/Mina/Wampfler/de Saussure/ Pautex 


\section{Acknowledgment}

We wish to acknowledge the nurses, nurse-aids, and other personnel of "Les Tilleuls" for the help in data collection and their practical advice. We also thank the families of the patients who accepted the prescription of a new medication to their family member, as well as Aurélie Revol and Sandro Cattacin (Faculty of Social Sciences, University of Geneva) for the social part of the study (to be published separately).

\section{Statement of Ethics}

The parents or guardians of the subjects gave their written informed consent for the prescription of the cannabinoids-based medication and for participation in the study. The study protocol was approved by the Geneva Canton Central Committee on $\mathrm{Hu}$ man Research (protocol number 2017-01196).

\section{Disclosure Statement}

The authors have no conflicts of interest to declare.

\section{Funding Sources}

An anonymous donor without any connections with the pharmaceutical industries paid the different medications provided for this study. The authors did not receive any financial compensation for their work.

\section{Author Contributions}

Prof. S. Pautex and Prof. B. Broers elaborated the research protocol, the request to the Ethics Commission, supervised the data collection and the master thesis of the two medical students, and wrote the draft of this article. Z. Patà and A. Mina, medical students, helped with the data collection, filled in the clinical record files, and made the database and data analysis under supervision. Dr. C. de Saussure and Director J. Wampfler initiated the first prescription of nabiximol and made the whole study possible from a practical point of view (contact with the families, requests to Federal Office of Public Health, prescriptions, safety issues).

\section{References}

1 WHO. Dementia [Internet]. WHO. 2017 [cited November 17, 2018]. Available from: http://www.who.int/mediacentre/factsheets/ fs $362 / \mathrm{en} /$.

2 Abraha I, Rimland JM, Trotta FM, Dell'Aquila G, Cruz-Jentoft A, Petrovic M, et al. Systematic review of systematic reviews of non-pharmacological interventions to treat behavioural disturbances in older patients with dementia. The SENATOR-OnTop series. BMJ Open. 2017 Mar;7(3):e012759.
3 Aso E, Ferrer I. Cannabinoids for treatment of Alzheimer's disease: moving toward the clinic. Front Pharmacol. 2014 Mar;5:37.

4 Szeto JY, Lewis SJ. Current Treatment Options for Alzheimer's Disease and Parkinson's Disease Dementia. Curr Neuropharmacol. 2016;14(4):326-38.

5 Mina A, Pata Z. Prescription de cannabinoïdes en Etablissement Médico-Social. Thèse de maîtrise, Faculté de Médecine, Université de Genève, juin 2017.

6 Krishnan S, Cairns R, Howard R. Cannabinoids for the treatment of dementia. Cochrane Database Syst Rev. 2009 Apr;(2): CD007204.
7 Cummings JL, Mega M, Gray K, RosenbergThompson S, Carusi DA, Gornbein J. The Neuropsychiatric Inventory: comprehensive assessment of psychopathology in dementia. Neurology. 1994 Dec;44(12):2308-14.

8 Robert PH, Vincent S, Staccini PC, Goni S L'inventaire neuropsychiatrique: validation de la version française d'un instrument destiné à évaluer les troubles du comportement chez le sujet dément. Annee Gerontol. 1998;5: 63. 